\title{
CISTECTOMÍA EN TUMORES VESICALES SUPERFICIALES. ANÁLISIS DE LOS CRITERIOS DE INCLUSIÓN Y FACTORES PRONÓSTICOS
}

\author{
N. ALONSO GRACIA, J.A. LORENTE GARÍN, E. DE LEÓN MORALES, \\ D. CAÑÍS SÁNCHEZ, O. BIELSA GALÍ, A. GELABERT-MAS
}

Servicio y Cátedra de Urología. Hospital del Mar. UAB. Barcelona.

Actas Urol Esp. 27 (5): 350-355, 2003

\section{RESUMEN}

"CISTECTOMÍA EN TUMORES VESICALES SUPERFICIALES. ANÁLISIS DE LOS CRITERIOS DE INCLUSIÓN Y FACTORES PRONÓSTICO"

INTRODUCCIÓN: Aunque el tratamiento estándar del tumor vesical superficial es la resección transuretral, excepcionalmente y por diversas circunstancias clínicas se puede indicar cistectomía radical en estos pacientes.

PACIENTES Y MÉTODO: Entre junio de 1986 y diciembre de 2001 se realizaron 127 cistectomías radicales. Se analizó una serie de 25 pacientes con cistectomía radical realizada en tumores vesicales superficiales. Se analizaron las indicaciones, la correlación anatomo-patológica y la evolución clínica con las curvas de supervivencia.

RESULTADOS: El tiempo medio de seguimiento fue de 47 meses. La indicación principal fueron los tumores GIIIpT1 extensos (40\%). El resto de la serie incluía carcinomas no controlables endoscópicamente y refractarios a la quimioterapia. Existió coincidencia en la correlación anatomo-patológica en el $48 \%$ de los pacientes observándose supra e infraestadiaje en el $25 \%$ y $28 \%$ respectivamente. El periodo libre de recidiva fue de 54 meses. En el seguimiento fallecieron siete pacientes (28\%) mientras 18 pacientes $(72 \%)$ siguen vivos sin enfermedad. La media de la supervivencia actuarial fue de 128,48 meses.

CONCLUSIONES: Los tumores GIIIpT1 fueron la indicación principal de nuestra serie. Destaca el porcentaje significativo de infraestadiaje, la baja morbilidad y las buenas curvas de supervivencia que se obtienen. Estos resultados nos permiten considerar a la cistectomía radical como una alternativa viable en pacientes seleccionados con tumor vesical superficial.

PALABRAS CLAVE: Cistectomía radical. Tumor vesical superficial. Infraestadiaje.

\section{ABSTRACT \\ "CISTECTOMY IN SUPERFICIAL BLADDER TUMOURS. ANALYSIS OF CRITERION INCLUDE AND PREDICTION FACTORS"}

INTRODUCTION: Though usually the treatment of a superficial bladder tumour consists in transurethral resection, exceptionally because of several clinical conditions, in this kind of patients a radical cystectomy may be suitable.

PATIENTS AND METHOD: From june 1986 through december 2001, 127 radical cystectomies were perfomed. A series of 25 patients with a radical cystectomy performed in superficial bladder tumours were analysed. Indications, anatomo-pathological correlation and clinical evolution with survival curves are analysed.

RESULTS: The mean time of follow-up was 47 months. Extensive GIIIpT1 tumours (40\%) were the chief indication. The remaining of the series consisted in carcinoma uncontrollable by endoscopy and refractary to chemotherapy. Anatomo-pathological correlation coincided in $48 \%$ of patients, existing supra and understaging in $25 \%$ and $28 \%$ respectively. A period of 54 months was without relapse. Seven patients died during the evolution (28\%), and 18 patients are alive without disease. 128,48 months were the mean actuarial survival.

CONCLUSIONS: GIIIPT1 tumours were the chief indication of our series. The significant percentage of understaging, poor morbidity and good survival curves are emphasized. Because of these results we consider that radical cystectomies are a viable choice for selected patients with superficial vesical tumour.

KEY WORDS: Radical cystectomy. Superficial vesical tumour. Understaging. 
$\mathrm{S}_{\mathrm{t}}^{\mathrm{e}}$ trata de un tema controvertido, ya que el ratamiento estándar del tumor vesical superficial es la resección transuretral (RTU), pero excepcionalmente por distintas circunstancias clínicas, el urólogo puede indicar la cistectomía radical en pacientes seleccionados. Del total de la incidencia de carcinoma urotelial vesical, el $75 \%$ corresponden a tumor superficial. De ellos sólo el 10-15\% progresarán con invasión de la capa muscular ${ }^{5,6,7,13}$. La mayoría de estos tumores superficiales se tratan con RTU más quimioterapia o inmunoterapia endovesical. No obstante existe un subgrupo con ciertas características que le confieren mayor agresividad. En estos casos seleccionados puede estar indicada la cistectomía.

Para evaluar la eficacia de esta actitud terapéutica, revisamos una serie de 25 pacientes con tumor vesical superficial de vejiga en los que se realizó cistectomia radical más linfadenectomía pélvica bilateral. Este estudio evalúa el aspecto clínico-patológico de los resultados obtenidos y la evolución clínica a medio y largo plazo.

\section{PACIENTES Y MÉTODO}

Se han realizado 127 cistectomías en nuestro servicio desde junio de 1986 a octubre del 2001.

Dentro de esta serie se realizó un estudio descriptivo-retrospectivo de un grupo de 25 pacientes $(19,6 \%)$ en los que se ha llevado a cabo una cistectomía en estadios Ta, T1 y CIS. En todos ellos se ha realizado una derivación urinaria tipo Bricker. La serie la forman una mujer (4\%) y 24 varones (96\%). La edad media es de 66 años (rango 53-77 años). La información la obtuvimos del historial médico.

En todos ellos se realizó RTU como primera maniobra terapéutica previa a la cirugía radical. Se revisó el porcentaje de pacientes que recibieron quimioterapia o inmunoterapia endovesical adyuvante. Se realizó un TAC en aquellos pacientes con sospecha macroscópica endoscópica de tumor invasivo.

Las indicaciones para realizar la cistectomía radical en estas circunstancias fueron: los carcinomas transicionales GIIIpT1 extensos (pantumores) y GIIIpT1 con fracaso de la inmunoterapia endovesical con BCG, carcinomas transicionales GI, GII superficial no controlable endoscópica- mente y que precisaban múltiples tiempos de RTU sin conseguir su control completo, carcinoma transicional refractario a quimioterapia endovesical que presentaba múltiples recidivas y un último grupo estaba formado por un paciente con carcinoma escamoso de vejiga

En el estudio valoramos la correlación anatomo-patológica, tanto del estadiaje como del grado citológico, entre la RTU y la cistectomía. En la clasificación anatomo-patológica se empleó el sistema TNM. La evolución clínica de los pacientes también fue revisada, incluyendo: la morbilidad operatoria, el periodo libre de enfermedad y la incidencia de recidiva de enfermedad urotelial. Se calcularon por el método Kaplan y Meier, las curvas de supervivencia actuarial global a 5 años y la supervivencia actuarial comparada del grupo de tumores infiltrantes frente al resto.

\section{RESULTADOS}

El tiempo medio de seguimiento de la serie fue de 47 meses (rango 1-186).

El tiempo medio de duración del tratamiento previo a la cistectomía fue de 8,16 meses (rango 0-51). Durante este tiempo la media de RTU de tumor vesical previo a la cirugía radical, fue de 1,76 RTU por paciente (rango 1-5). La media de tratamientos endovesicales fue de 0,64 por paciente (rango 0-3).

La distribución de los pacientes por criterio de inclusión fue la siguiente: en 10 pacientes (40\%) la indicación de cistectomía radical fue por tumor GIIIpT1, incluyendo tanto los extensos como los que fracasaron a la inmunoterapia; en 9 pacientes (36\%) la indicación fue por tumor no controlable endoscópicamente, en 5 pacientes $(20 \%)$ por tumor refractario a la quimioterapia o inmunoterapia endovesical con múltiples recidivas y en un paciente (4\%) por carcinoma escamoso (Gráfica I).

La correlación anatomo-patológica entre la RTU y la cistectomía, en 12 pacientes (48\%) coincidió el mismo estadiaje, en 6 pacientes (25\%) observamos un supraestadiaje y 7 pacientes (28\%) resultaron infraestadiados en la RTU (Chicuadrado=1780; g.1.=3; p=0,619) (Tabla I). En 4 pacientes del grupo de infraestadiados (57\%), el resultado definitivo fue de carcinoma transicional infiltrante, 2 pacientes (50\%) tenían inicialmente 


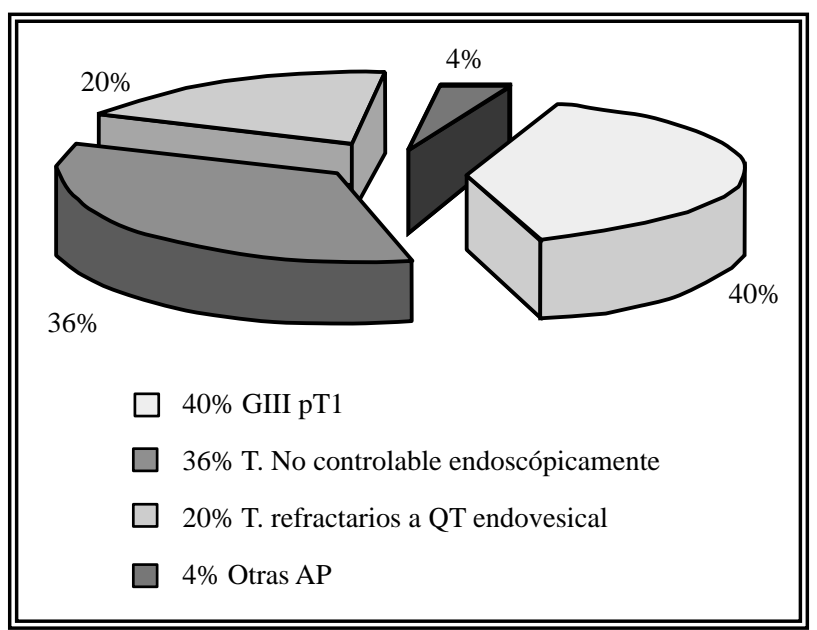

Gráfica I. Porcentaje y tipo de pacientes incluidos en cada indicación.

\section{TABLA I}

CORRELACIÓN ANATOMO-PATOLÓGICA DEL ESTADIAJE ENTRE LA RTU Y LA CISTECTOMÍA

\begin{tabular}{||l|c|c|}
\hline & $\begin{array}{c}\text { RTU } \\
\text { Ta }\end{array}$ & $\begin{array}{c}\text { RTU } \\
\text { T1 }\end{array}$ \\
\hline Cistectomía To & 0 & 1 \\
\hline Cistectomía Ta & 5 & 5 \\
\hline Cistectomía T1 & 3 & 7 \\
\hline Cistectomía & 1 & 3 \\
\hline
\end{tabular}

GIIIpT1 y 2 pacientes (50\%) presentaban tumores no controlables endoscópicamente. Respecto al grado de diferenciación citológico se analizaron 24 pacientes debido a que un paciente no presentó tumor en la pieza de cistectomía. En 19 pacientes $(79,1 \%)$ coincidió el mismo, en 3 pacientes $(12,5 \%)$ el grado de diferenciación de la RTU fue mayor y en 2 pacientes $(8,3 \%)$ el grado de diferenciación fue inferior (Chi-cuadra$\mathrm{do}=11,08 ;$ g.l. $=2 ; \mathrm{p}=0,004)$ (Tabla II).

Respecto a la evolución clínica, dos pacientes (8\%) requirieron ingreso en la unidad de cuidados intensivos en el post-operatorio inmediato, debido al empeoramiento de su patología respiratoria, un paciente fue éxitus por esa causa. El periodo libre de recidiva fue de 54 meses. Ningún paciente presentó recidiva pélvica. Dos pacientes (8\%) presentaron implantes en uretra bulbar por lo que se les practicó uretrectomía y en un
TABLA II

CORRELACIÓN ANATOMO-PATOLÓGICA DEL GRADO CITOLÓGICO ENTRE RTU Y CISTECTOMÍA

\begin{tabular}{||l|c|c|c||}
\hline & $\begin{array}{c}\text { RTU } \\
\text { Grado I }\end{array}$ & $\begin{array}{c}\text { RTU } \\
\text { Grado II }\end{array}$ & $\begin{array}{c}\text { RTU } \\
\text { Grado III }\end{array}$ \\
\hline Cistectomía Grado I & 0 & 0 & 0 \\
\hline Cistectomía Grado II & 1 & 9 & 3 \\
\hline Cistectomía Grado III & 0 & 1 & 10 \\
\hline
\end{tabular}

paciente (4\%), se evidenció tumor en el tracto urinario superior realizándose nefroureterectomía. En la actualidad, la serie refleja 7 pacientes fallecidos (28\%): 2 pacientes (8\%) fueron éxitus causa específica, y 5 pacientes (20\%) fueron éxitus no causa específica (un paciente (4\%) con enfermedad y 4 pacientes (16\%) libres de enfermedad). El resto de la serie lo forman 18 pacientes $(72 \%)$ que en la actualidad están vivos sin enfermedad.

Referente a la supervivencia cáncer específica, la media de la supervivencia actuarial fue de 128,48 meses. En cortes transversales de la curva de Kaplan y Meier, a 12 meses la supervivencia actuarial fue de $96 \%$, a 30 meses fue de $74,67 \%$ y a 60 meses de $63,56 \%$ (Gráfica II). La probabilidad de supervivencia actuarial en los pacientes con estadios T2 o más (grupo de los 7

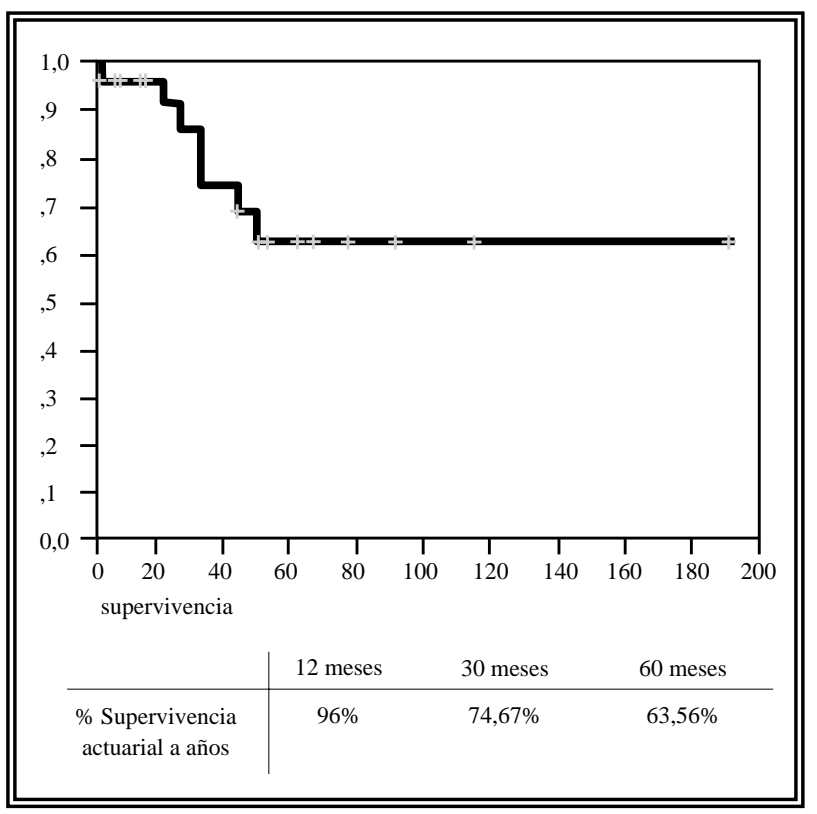

GRÁFICA II. Curva de Kaplan y Meier de supervivencia global actuarial a 5 años 


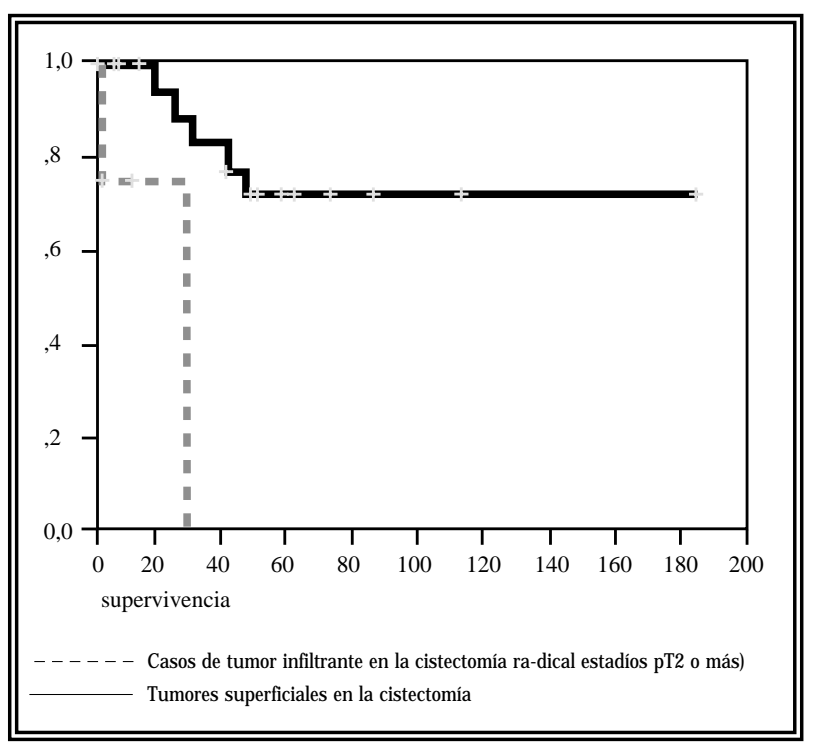

GRÁFICA III. Curvas de Kaplan y Meier de la probabilidad estimada de supervivencia global de los casos de tumor vesical superficial en la pieza de cistectomía frente a los casos cuyo resultados en a anatomía patológica definitiva fue infiltrante.

pacientes infraestadiados), fue significativamente peor que la supervivencia del resto de la serie $(\mathrm{p}=0,0024)$ (Gráfica III).

\section{DISCUSIÓN}

Es aceptado de manera estándar, que la cistectomía radical más linfadenectomía pélvica bilateral es el tratamiento de elección del tumor urotelial de vejiga invasivo, y que el tratamiento endoscópico debe aplicarse para el tumor urotelial que no sobrepasa la lámina propia. Pero para ambas afirmaciones existen autores que defienden distintas posturas, ya que algunos autores presentan resultados similares en tumores uroteliales $\mathrm{T} 2$ para el tratamiento endoscópico versus cistectomía radi$\mathrm{cal}^{20-22}$. Cabe destacar que existen grupos de trabajo, que buscando un tratamiento endoscópico más completo y así evitar la indicación de cistectomía, presentan buenos resultados con una aceptable tolerabilidad de la terapia fotodinámica ${ }^{15}$.

El papel de la cistectomía radical en el tumor urotelial de vejiga superficial no está bien definido. Debe ponerse en la balanza el posible sobretratamiento, con el riesgo y las consecuencias que la cirugía conlleva y la posibilidad de un tratamiento efectivo durante un periodo de potencial curabilidad.
La historia natural de la mayoría de tumores superficiales refleja una respuesta correcta tras la RTU más quimioterapia o inmunoterapia endovesical. Hasta el momento es difícil identificar, aquellos tumores que van a progresar, a pesar de los múltiples estudios realizados tanto clínicos, como con marcadores moleculares. Los estudios con marcadores moleculares no permiten hasta la fecha ser utilizados como factor único en la decisión de realizar una cistectomía precoz ${ }^{9}$. Las características de los tumores que se asocian con capacidad para infiltrar la capa muscular han sido descritas en múltiples series ${ }^{1-5,7,8}$. La invasión de la lámina propia, invasión vascular o linfática de la lámina propia, tumor de alto grado, multifocalidad y asociación con CIS, son los factores clásicamente manejados como los que confieren mayor agresividad a las lesiones superficiales. Múltiples recidivas a pesar de la quimioterapia o inmunoterapia endovesical profiláctica también sugieren mayor agresividad ${ }^{3}$. Estos factores, más los problemas endoscópicos de control del tumor, plantean al urólogo una duda razonable acerca de la viabilidad del tratamiento conservador en algunos casos.

Basándose en estas circunstancias algunos autores defienden la indicación de cistectomía precoz en estos pacientes.

Haciendo referencia a los tumores de gran volumen, no existe consenso en su definición. Algunos autores ponen el límite en $3 \mathrm{~cm}$. y otros en 5 gr. En unas ocasiones el elevado volumen se consigue sumando los múltiples tumores resecados, y en otras, por un tumor de gran volumen que ocupa más del $80 \%$ de la superficie. Esta entidad es conocida coloquialmente como papilomatosis vesical (Fig. 1). Estos datos hacen difícil su definición y la comparación entre las distintas series ${ }^{18}$. Nuestro criterio de inclusión se basó en aquellos tumores, en los cuales, 2 o más tiempos de RTU en manos expertas no permitían conseguir su control. El porcentaje sobre el total de cistectomías realizadas en ese mismo periodo fue de $7,8 \%$, similar al de otras series ${ }^{18}$. Mención especial requiere el tumor GIIIpT1 como indicación principal en nuestra serie, en la que se incluyeron aquellos que abarcaban una amplia zona de la mucosa y por estadiaje clínico se suponía infiltrante, y aquellos extensos que tras tratamiento 


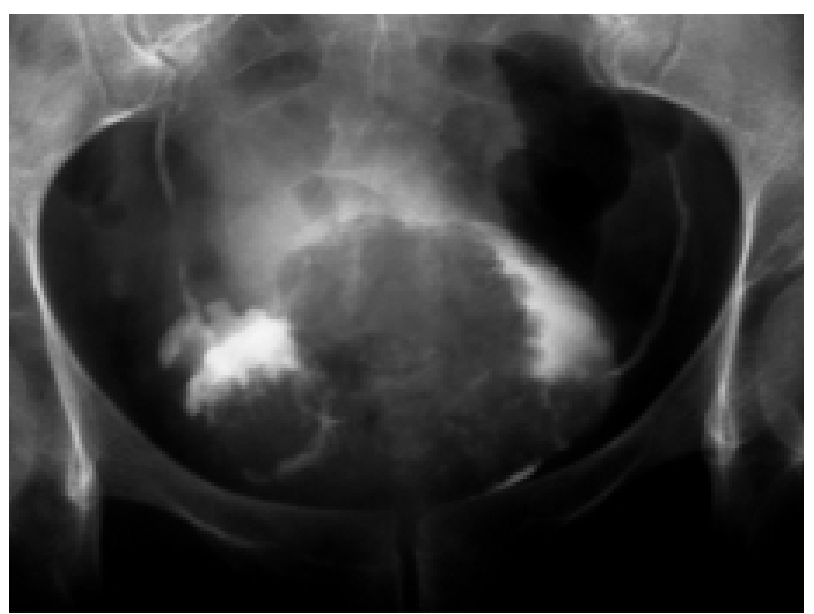

FIGURA 1. Papilomatosis vesical.

endovesical con BCG, en una segunda RTU de reestadiaje persistía el tumor. Hemos comentado previamente que la invasión de la lámina propia y el alto grado confieren por sí solos mayor agresividad a las lesiones superficiales, pero los buenos resultados obtenidos con la BCG intravesical, con tasa de respuesta completa del 50-70\% ${ }^{9}$, dejan en segundo plano a la cistectomía radical como tratamiento inicial de elección en los tumores GIIIpT1. Incluso Zungri y cols. presentan en su última serie, índices de progresión del 23,5\% en GIIIpT1 sin tratamiento adjuvante, siendo un porcentaje más bajo que los publicados anteriormente por otros autores ${ }^{16}$. Existen sin embargo ciertos factores pronósticos que inclinarían la decisión, en algunos casos de tumores GIIIpT1, hacía una cistectomía precoz. El fracaso de la terapia intravesical está asociado a un porcentaje significativo de infraestadiaje y de metástasis, afectación de la uretra prostática, multifocalidad y nivel de invasión en profundidad de la lámina propia $^{4,9,11}$. Solsona y cols. recomiendan la cistectomía radical en aquellos tumores GIIIpT1 multifocales asociados a CIS, tanto en el diagnóstico inicial como en su seguimiento ${ }^{17}$.

La incidencia de infraestadiaje en pacientes con tumores clínicamente superficiales es significativo $^{8}$. En nuestra serie se comunican cifras de infraestadiaje del 28\%. Ninguno de los pacientes infraestadiados presentó enfermedad extravesical $(\mathrm{T} 4, \mathrm{~N}+)$. Distintas series aportan porcentajes de infraestadiaje muy similares que rondan el $30 \%{ }^{1,5,10}$. Freeman y cols. refieren en su serie un
$34 \%$ de $\mathrm{T} 2 \mathrm{~N}+\mathrm{M}+$ en tumores presumiblemente superficiales y defienden la dificultad de detectar la invasión previamente a la cistectomía ${ }^{8}$. Dutta y cols. aportan valores algo superiores con un 40\%, e indican que la ausencia de muscular propia en la biopsia es un factor de riesgo significativo para el infraestadiaje ${ }^{7}$. Cabe destacar que el 50\% de los tumores que resultaron infiltrantes en nuestra serie eran inicialmente GIIIpT1. Esto implica que el 20\% de los GIIIpT1 a los que se les indicó una cistectomia ya habian progresado. Huguet J. y cols. aportan porcentajes similares en su serie, con el 27,5\% de progresión en los GIIIpT1 en la pieza de cistectomía ${ }^{10}$. Estos datos deberían ponernos en alerta frente aquellos superficiales de alto grado en los que tenemos duda acerca de su control endoscópico. Respecto al grado citológico la mayoría de autores coincide que es el principal factor pronóstico de progresión y mortalidad. Tomando como referencia el grado I, el grado II y el grado III tienen un riesgo relativo 3 y 20 veces mayor respectivamente ${ }^{19}$. En nuestra serie la correlación entre el grado citológico de la RTU y la cistectomia fue significativo al igual que presentan Cheng y cols. en su serie ${ }^{14}$. Esto implicaría que es infrecuente tanto la infra como la supragradación.

En cuanto a la supervivencia, como se esperaba, la curva actuarial en el grupo de tumores que en la pieza definitiva resultaron infiltrantes, fue significativamente peor $(\mathrm{p}<0,01)$ respecto al resto de la serie. Similares resultados reportan otros estudios $^{1,5,7,8}$.

La preocupación por la alteración de la calidad de vida que se le atribuye a la cistectomía radical más la derivación urinaria, ha disminuido con el desarrollo de las técnicas de derivación continente y la reconstrucción del tracto urinario inferior, tanto para varones como para mujeres, reduciendo las implicaciones psicosociales del conducto ileal ${ }^{5,8}$. Asociado a la derivación continente ortotópica, la cistectomía radical con preservación neural podría representar el mejor abordaje quirúrgico $^{13}$. Existen autores que trabajan sobre nuevas técnicas quirúrgicas que diminuyan la morbilidad post-operatoria. Colombo R. y cols. realizan en una serie de pacientes jóvenes con tumor vesical superficial, un primer tiempo de RTU de próstata seguido de linfadenectomía pélvica, cistectomía extraperitoneal preservando los 
deferentes, bandeletas neurovasculares y un reservorio ileal W-shaped anastomosado a la cápsula prostática. En su serie mantienen una buena calidad de vida, preservando la potencia y la fertilidad ${ }^{12}$.

Con todos estos hallazgos, destacando el porcentaje significativo de infraestadiaje, la baja morbilidad y las buenas curvas de supervivencia que se obtienen, permiten considerar a la cistectomía radical más derivación urinaria, una buena opción en aquellos tumores uroteliales de vejiga con características que le confieren agresividad; y que exponen a un riesgo potencial de progresión de estadio, de invasión ganglionar, de metástasis y muerte.

\section{REFERENCIAS}

1. MALKOWICZ SB, NICHOLS P, LIESKOVSKY G, BOYD SD, HUFFMAN J, SKINNER DG: The role of radical cystectomy in the management of high grade superficial bladder cancer (PA, P1, PIS and P2). J Urol 1990; sep. 144: 641-645.

2. ANDERSTRÖM C, JOHANSSON S, NILSSON S: The significance of lamina propia invasion on the prognosis of patiens with bladder tumors. J Urol 1980; 124: 23.

3. LUTZEYER W, RÜBBEN H, DAHM H: Prognosis parameters in superficial bladder cancer: an analysis of 315 cases. J Urol 1982; 127: 250.

4. ESRIG D, FREEMAN JA, STEIN JP, SKINNER DG: Early cystectomy for clinical stage $\mathrm{T} 1$ transitional cell carcinoma of the bladder. Semin Urol Oncol 1997; aug. 15 (3): 154-160.

5. AMLING CL, THRASHER JB, FRAZIER HA, DODGE RK, ROBERTSON JE, PAULSON DF: Radycal cystectomy for stages TA, TIS and T1 transitional cell carcinoma of the bladder. J Urol 1994; jan. 151: 31-36.

6. American Cancer Society: Bladder Cancer: In: Cancer Facts and Figures-1992: 14.

7. DUTTA SC, SMITH JA, SHAPPELL SB, COFFEY CS, CHANG SS, COOKSON MS: Clinical under staging of high risk nonmuscle invasive urothelial carcinoma treated with radical cystectomy. J Urol 2001; aug 166: 490-493.

8. FREEMAN JA, ESRIG D, STEIN JP et als.: Radycal cystectomy for high risk patients with superficial bladder cancer in the era of orthotopic urinary reconstruction. Cancer 1995; sep. 76 (5): 833-839.

9. GARCÍA M, RICO J, CAMACHO E: La cistectomía radical en el carcinoma vesical superficial . Actas Urol Esp 2001; may. 25 (5): 329-334.

10. HUGUET J, PALOU J, MILLÁN F, VILLAVICENCIO $\mathrm{H}$, VICENTE J: Cistectomía radical en tumores superficiales en la era de la BCG. Arch Esp Urol 2002; feb. 55 (1): 50-56.
11. SOLOWAY MS. Managing superficial bladder cancer: an overview. Suplement to Urology 1992; dec. 40 (6): 5-9.

12. COLOMBO R, BERTINI R, SALONIA A et al.: Nerve and seminal sparing radical cystectomy with orthotopic urinary diversion for select patientes with superficial bladder cancer: an innovative surgical approach. J Urol 2001; jan. 165 (1): 51-55.

13. VAN POPPEL H: La cistectomía en el tratamiento del cáncer superficial de vejiga. In: Vicente J, Chéchile G, Salvador J. Tumores vesicales superficiales. 1 ed. Acción Médica, S.A. 2000: 191-200.

14. CHENG L, NEUMANN RM, WEAVER AL et al.: Grading and staging of bladder carcinoma in transurethral resection specimen. Correlation with 105 matched cystectomy specimens. Am J Clin Pathol 2000; feb. 113 (2): 275-279.

15. SHACKLEY DC, BRIGGS C, GILHOOLEY A et al.: Photodynamic therapy for superficial bladder cancer under local anaesthetic. BJU Int 2002; may. 89 (7): 665-670.

16. ZUNGRI E, MARTÍNEZ L, DA SILVA EA, PESQUEIRA D, DE LA FUENTE A, PEREIRO B: T1GIII bladder cancer. Management with transurethral resection only. Eur Urol 1999; 36: 380-385.

17. SOLSONA E, IBORRA I, RICOS JV, MONROS JL, RUBIO J, ALMENAR S.: Clinical panurothelial disease in patients with superficial bladder tumors: therapeutic implications. J Urol 2002; may. 167 (5): 2007-2011.

18. CHÉCHILE GE: Tumores vesicales superficiales de gran volumen. In: Vicente J, Chéchile G, Salvador J. Tumores vesicales superficiales. 1 ed. Acción Médica, S.A. 2000: 279-283.

19. MILLÁN F: Factores pronósticos clínicos. In: Vicente J, Chéchile G, Salvador J. Tumores vesicales superficiales. 1 ed. Acción Médica, S.A. 2000: 33-45.

20. SOLSONA E, IBORRA I, RICOS JV, MONROS JL, CASANOVA J, CALABUIG C: Feasibility of transurethral resection for muscle infiltrating carcinoma of the bladder: long-term followup of a prospective study. J Urol 1998; jan. 159 (1): 95-98; discussion 98-99.

21. HERR HW: Transurethral resection of muscle-invasive bladder cancer: 10-year outcome. J Clin Oncol 2001; jan 1. 19 (1): 89-93.

22. PÁEZ A, LUJÁN M, ROMERO I, LLÁNES L, GÓMEZ JM, BERENGUER A: Preservación vesical selectiva mediante resección transuretral de los tumores músculo-infiltrantes. Actas Urol Esp 2001; apr. 25 (4): 264-268.

Dra. N. Alonso Gracia

Hospital del Mar. Servicio de Urología

Passeig Maritim, 25-29

08003-Barcelona

(Trabajo recibido el 27 de septiembre 2002) 